\title{
Cycle biologique de Rictularia proni Seurat, 1915 Nematoda Rictulariidae
}

\section{Ontogénèse des structures céphaliques}

\author{
par J.-C. QUENTIN \\ Station biologique F 34 - La Borie Nouvelle, \\ Laboratoire de Zoologie (Vers), associé au C.N.R.S. (Pr A.-G. ChABAud) \\ Muséum National d'Histoire Naturelle, 57, rue Cuvier, $75-$ Paris, $5^{\circ}$
}

\begin{abstract}
Résumé
Cycle biologique expérimental du Nématode Spiruride Rictularia proni Seurat, 1915 dont l'adulte parasite fréquemment le Rongeur Apodemus sylvaticus L.

Les trois premiers stades larvaires évoluent dans la paroi du proctodeum d'Orthoptères: Locusta migratoria L., Oedipoda germanica Latreille, Omocestus raymondi Yersin, de Dermaptère Forficula auricularia $\mathrm{L}$. et de Coléoptère Tenebrio molitor $\mathrm{L}$.

La croissance larvaire puis l'obtention des adultes mâles sont étudiées chez le Mulot. L'ontogénèse des structures céphaliques du Nématode permet de préciser, dès le début du quatrième stade larvaire, la séparation dans la famille des Rictulariidae des genres Rictularia et Pterygodermatites fondée précédemment sur les structures buccales des adultes.
\end{abstract}

\section{Summary}

Experimental biological cycle of the Spirurid Nematode Ric. tularia proni Seurat, 1915 which adult is known as a frequent parasit of the Rodent Apodemus sylvaticus L. 
The first three larval stages occur in the proctodeum wall of the Orihopteras Locusta migratoria L., Oedipoda germanica Latreille, Omocestus raymondi Yersin, the Dermaptera Forficula auricularia L. and the Coleoptera Tenebrio molitor L.

Larval growth and completion of adult stage occur in the Rodent.

From data on the ontogenesis of the cephalic structures of this Nematode it is now possible to separate the genus Rictularia from the genus Pterygodermatites inside the family Rictulariidae as early as the fourth larval stage such a distinction being formerly based upon the adult buccal apparatus only.

Les autopsies de 23 Apodemus sylvaticus L. à la station biologique de la Borie Nouvelle (Hérault), France, durant le mois de juiilet 1969, ont permis de récolter successivement dans l'intestin grêle de deux d'entre eux, 1 б 0,1 et $2 \uparrow$ gravides du Nématode Spiruride Rictularia proni Seurat, 1915.

\section{A. - Evolution chez L'Hôte intermédiatre.}

$1^{\circ}$ Infestation. L'infestation expérimentale de l'hôte intermédiaire est réalisée en apposant les œufs embryonnés du parasite sur les pièces buccales d'Insectes appartenant aux ordres suivants :

- Orthoptères (*): Locusta migratoria L. provenant du Vivarium du Museum Edipoda germanica Latreille et Omocestus raymondi Yers.n recueillis autour de la Station de la Borie Nouvelle.

- Dermaptère: Forficula auricularia L. récoltés dans l'Hérault et en Normandie.

- Coléoptère : Tenebrio molitor L. en élevage au laboratoire.

L'infestation réussit chez les cinq espèces d'Insectes. Le nombre de capsules de une à trois chez Forficula auricularia peut atteindre $142 \mathrm{chez}$ Locusta migratoria.

Chez tous les Insectes, les larves sont encapsulées en arrière des tubes de Malpighi dans la paroi du proctodeum et sont situées entre la membrane basale et la cuticule (Photos 1-2). Nous n'avons jamais observé de larves du $3^{e}$ stade dans l'hémocoele.

$2^{\circ}$ Allure du développement chez l'Insecte. L'évolution des stades larvaires est suivie en maintenant les Insectes à une température de $28^{\circ} \mathrm{C}$. Les larves sont observées trois jours, sept jours, neuf jours, onze jours, quinze jours, seize jours et quarante-six jours après l'infestation. L'évolution paraît semblable chez ces différentes espèces d'Insectes où les larves infestantes du Spiruride obtenues après la même durée de développement entrent en diapause.

Le troisième jour après l'infestation, de nombreux œufs et larves sont observés dans la lumière intestinale. L'œuf mesure $57 \times 43 \mu$ et renferme une larve repliée

(*) Les déterminations d'Insectes nous ont aimablement été communiquées par le $\mathrm{P}^{\mathrm{r}}$ Chopard. 
plusieurs fois sur elle-même (fig. 1-A). Celle-ci libérée est longue de 258 à $300 \mu$, large de 10 à $12 \mu$. Elle pénètre dans la paroi intestinale, atteint $306 \mu$ de long sur $18 \mu$ de large ; elle est encapsulée une semaine après l'infestation. Elle mesure alors $338 \mu$ de long sur $26 \mu$ de large.

C'est à cette période que survient la première mue. La larve du second stade se libère de son exuvie dans les régions céphalique et caudale; sa taille est de 338 à $380 \mu$.
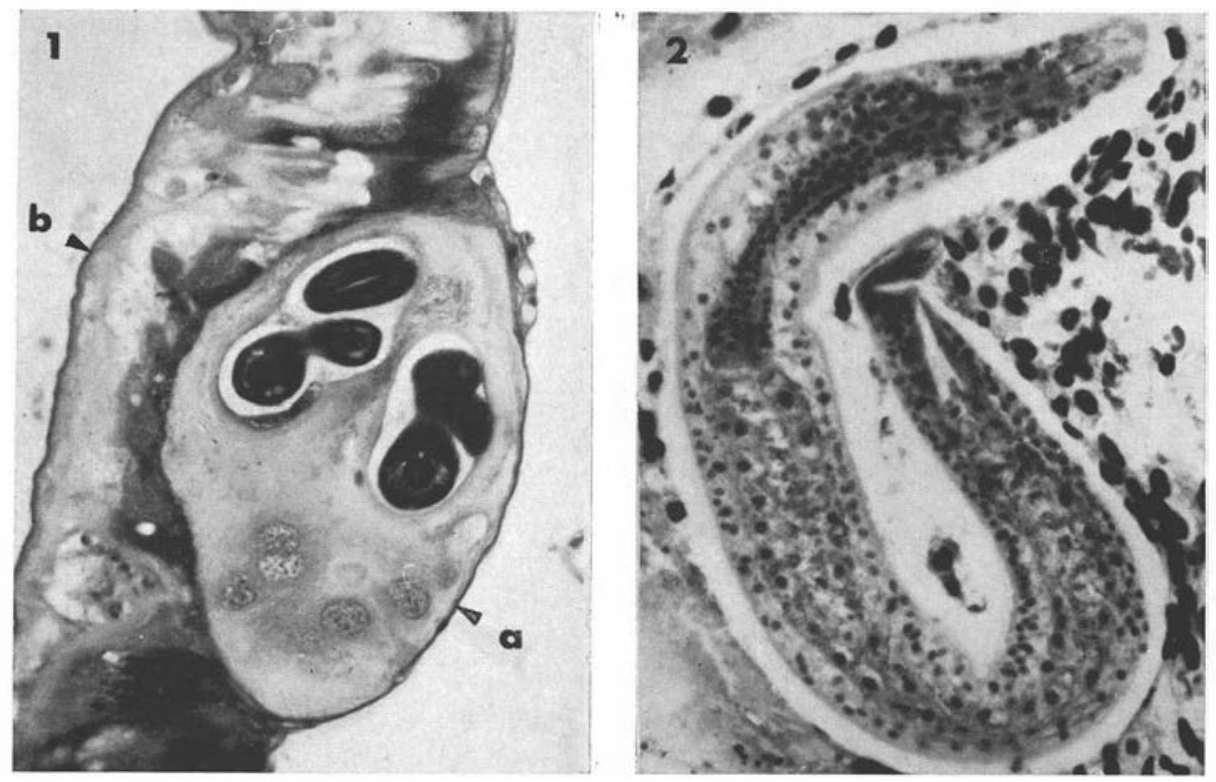

Pнотоs 1 et 2. - 1. Larves du $3^{\text {e }}$ stade de Rictularia proni encapsulées dans la paroi de l'intestin postérieur de l'Insecte entre la membrane basale $(a)$ et la cuticule $(b)$. 2. Larve du $3^{e}$ stade sectionnée longitudinalement au niveau de son tube digestif, coupe tangentielle de la paroi intestinale de l'Insecte

La croissance maximum, $550 \mu$, est atteinte neuf jours après l'infestation. La larve toujours du deuxième stade, contractée sur sa face dorsale est enfermée dans une capsule de $260 \mu$ de diamètre.

Les formations apicales du troisième stade apparaissent le onzième jour et la cuticule du second stade se décolle vers la pointe caudale.

Les larves âgées de quinze jours sont au troisième stade. Elles sont remarquables par leur faible taille 430 à $520 \mu$.

$3^{\circ}$ Morphologie des stades larvaires chez l'Insecte. L'évolution des structures céphaliques des différents stades est détaillée dans un chapitre particulier, nous ne décrirons ici que la morphologie générale. 


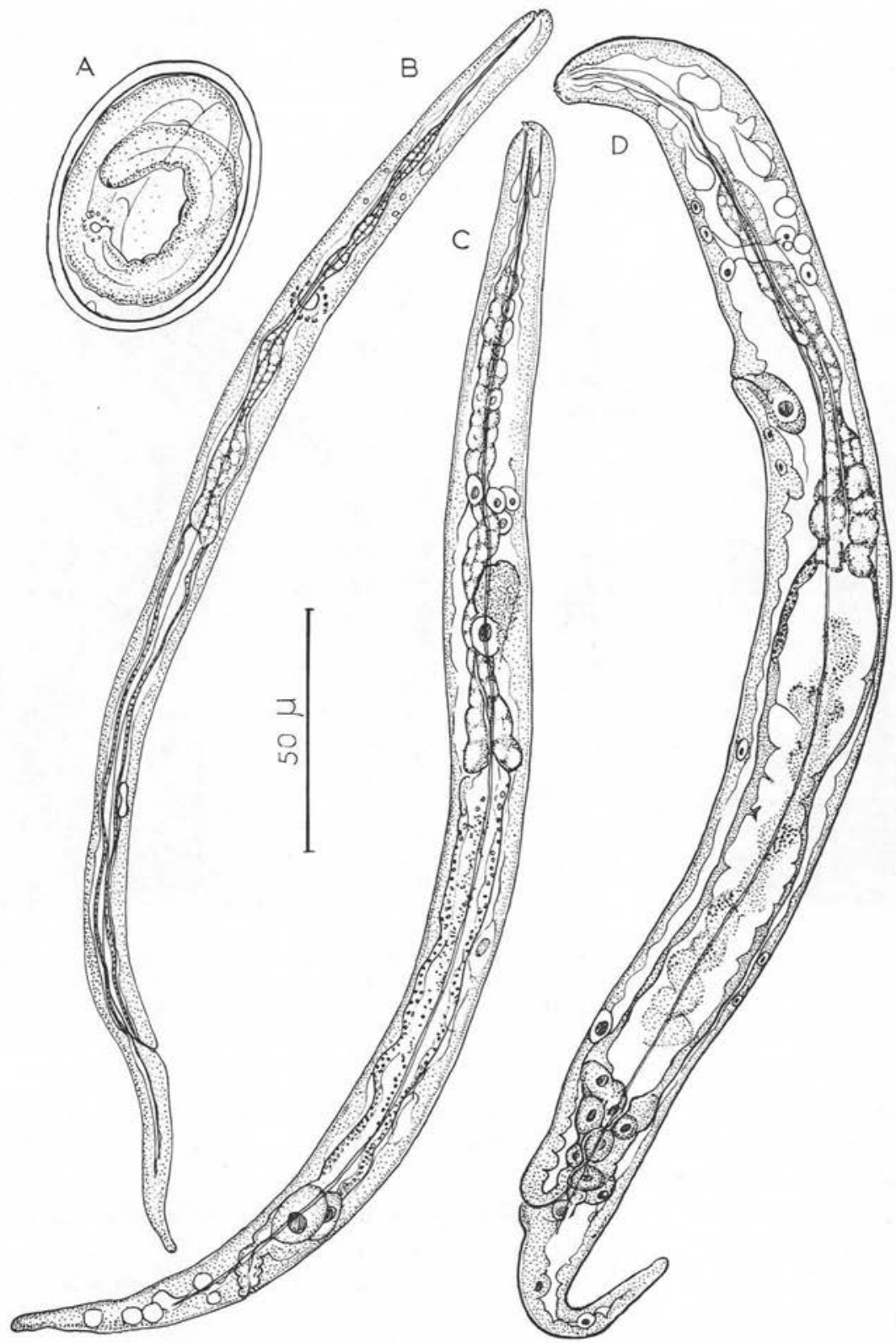

Fig. 1. - $1^{\text {er }}$ stade larvaire : A : œuf embryonné ; B : larve du $1^{\text {er }}$ stade, vue latérale dans la lumière intestinale de l'Insecte; $\mathrm{C}$ : larve du $1^{\text {er }}$ stade, vue latérale. Cette larve non encapsulée a pénétré dans la paroi intestinale; D : larve à la fin du $1^{\mathrm{er}}$ stade encapsulée dans la paroi intestinale A, B, C, D : éch. $50 \mu$ 
Premier stade: Deux très fines ailes latérales parcourent la larve sur sa longueur (fig. 1). Les râpes cuticulaires céphaliques habituelles aux premiers stades des Spirurides n'ont pas été observées.

L'épine céphalique est en retrait par rapport à l'apex. Les glandes céphaliques sont peu visibles. Par contre, la cellule excrétrice est réfringente et entourée de canalicules radiaires. La partie œsophagienne du tube digestif est mince et ne s'épaissit dans sa région postérieure qu'à la fin du premier stade.

Les dimensions d'une larve longue de $285 \mu$ (fig. 1-B) sont les suivantes: largeur $12 \mu$, cellule excrétrice située à $80 \mu$ de l'apex, longueur totale de l'œsophage $135 \mu$, de l'intestin $114 \mu$, ébauche génitale située à $190 \mu$ de l'extrémité antérieure, longueur de la queue $50 \mu$.

Les dimensions de la larve non encapsulée dans la paroi de l'intestin (fig. 1-C) sont: longueur $306 \mu$, largeur $18 \mu$, anneau nerveux et pore excréteur situés à 80 et $97 \mu$ de l'apex, longueurs respectives du préœsophage, de l'œsophage et de l'intestin, $33 \mu, 106 \mu$ et $125 \mu$, ébauche génitale située à $177 \mu$ de l'apex, queue longue de $52 \mu$.

A la fin du premier stade (fig. 1-D), la larve encapsulée mesure $338 \mu$ de long et 28 is de large. L'anneau nerveux et le pore excréteur sont situés à 57 et $78 \mu$ de l'apex. Les longueurs respectives du préœsophage, de l'œsophage et de l'intestin sont $50 \mu, 87 \mu$ et $150 \mu$. La cellule génitale est située à $250 \mu$ de l'apex, la queue mesure $55 \mu$.

Deuxième stade: La taille des larves du second stade âgées de sept jours est comprise entre 338 et $380 \mu$. Ces larves sont caractérisées par la formation du vestibule buccal et de l'œsophage musculaire, par la condensation des cellules autour de l'anneau nerveux et la présence d'une volumineuse cellule excrétrice.

Les cellules de l'ébauche génitale sont au nombre de quatre à six.

La larve longue de $380 \mu$ (fig. 2-A) a une largeur de $25 \mu$. L'anneau nerveux et le pore excréteur sont situés respectivement à 70 et $97 \mu$ de l'apex. La partie musculaire de l'œsophage est longue de $57 \mu$. La région glandulaire plus renflée mesure $74 \mu$. L'ébauche génitale est située à $230 \mu$ de l'apex. La queue mesure $60 \mu$.

Au neuvième jour du développement (fig. 2-B), la larve atteint sa taille maximum.

Ses dimensions sont les suivantes : $548 \mu$, largeur, $52 \mu$, anneau nerveux et pore excréteur à $85 \mu$ et $100 \mu$ de l'apex, vestibule buccal profond de $18 \mu$, longueurs respectives de l'œsophage musculaire, de l'œsophage glandulaire et de l'intestin, $70 \mu, 100 \mu$ et $304 \mu$, longueur de la queue $68 \mu$.

Les cellules génitales sont disposées sur une longueur de $52 \mu$ à une distance de l'apex de $370 \mu$.

Au onzième jour de l'évolution, chez l'Insecte, la capsule buccale large de $13 \mu$ profonde de $10 \mu$, du stade infestant est ébauchée autour du vestibule. L'aile latérale non encore striée du troisième stade apparaît par transparence. 


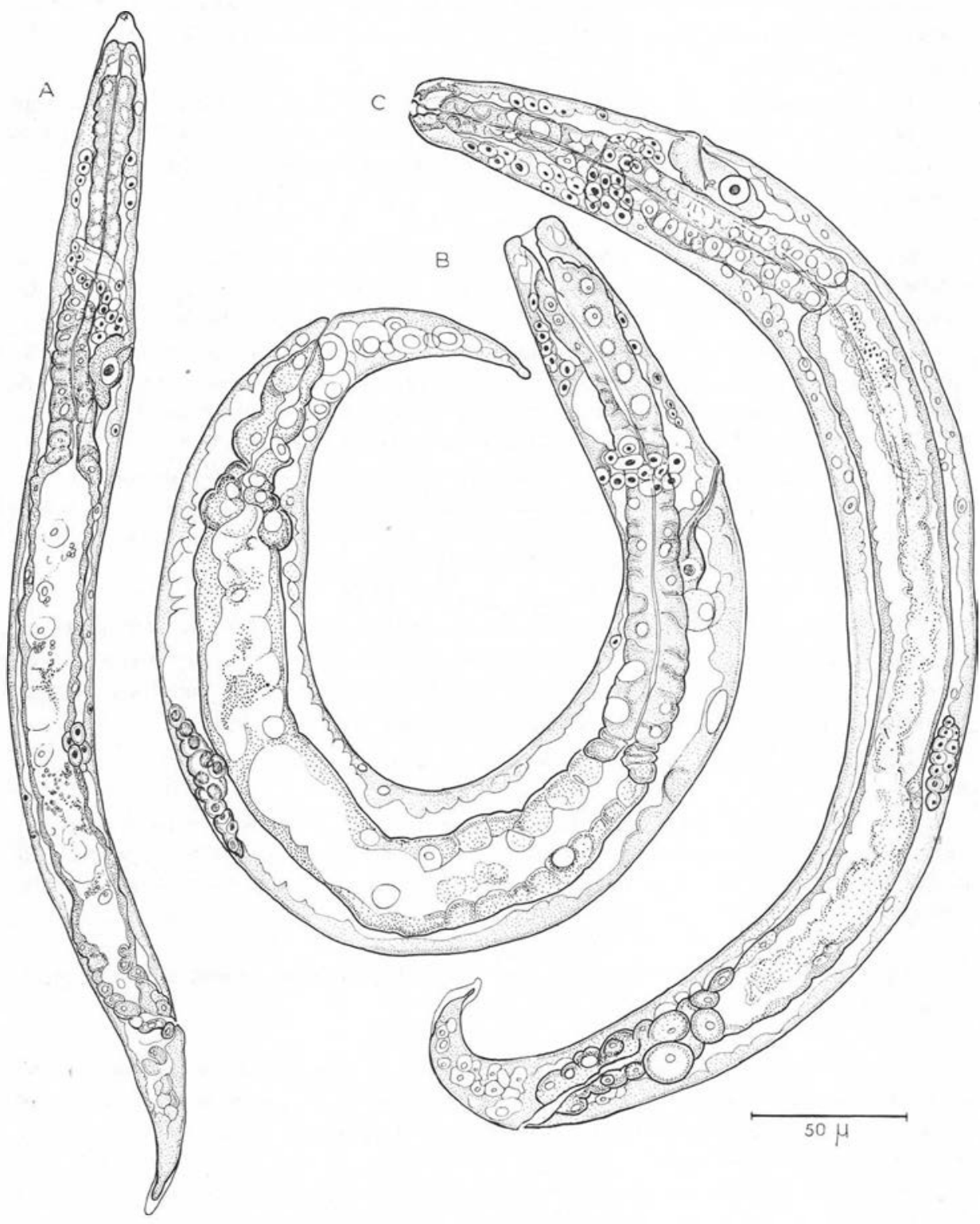

FIG. 2. $-2^{\mathrm{e}}$ stade larvaire: A : larve au début du second stade enfermée dans l'exuvie du $1^{\mathrm{er}}$ stade ; B : larve au $9^{\circ}$ jour de l'évolution chez l'Insecte $\left(\mathrm{T}^{\circ} 28^{\circ} \mathrm{C}\right) ; \mathrm{C}$ : larve fin du second stade, les structures céphaliques du $3^{\mathrm{e}}$ stade sont ébauchées, la cuticule est décollée dans la région caudale Les trois larves sont en vue latérale. A, B, C: éch. $50 \mu$ 
Cette larve (fig. 2-C) présente les dimensions suivantes: longueur $530 \mu$, largeur $43 \mu$, anneau nerveux et pore excréteur situés respectivement à 70 et $94 \mu$ de l'apex, longueurs respectives de l'œsophage musculaire, de l'œsophage glandulaire et de l'intestin, $47 \mu, 108 \mu$ et $295 \mu$, longueur de la queue, $55 \mu$.

Les cellules génitales forment un amas localisé à $322 \mu$ de l'apex.

Troisième stade larvaire: Les larves du troisième stade sont caractérisées par leur contraction dorsale, leur denticulation céphalique, l'apparition des deirides et des ailes latérales striées. L'extrémité caudale devient plus pointue.

Les dimensions relevées sur une larve de $496 \mu$ de long (fig. 3-A) sont: largeur $41 \mu$, anneau nerveux, pore excréteur et deirides respectivement situés à $70 \mu, 98 \mu$ et $98 \mu$ de l'apex, capsule buccale profonde de $13 \mu$, large de $10 \mu$ garnie de trois dents esopha. giennes denticulées à leur apex, longueurs respectives de l'œsophage musculaire, de l'œsophage glandulaire et de l'intestin, $50 \mu, 110 \mu$ et $265 \mu$, longueur de la queue, $60 \mu$.

Les cellules génitales forment un amas long de $55 \mu$ disposé à $286 \mu$ de l'apex.

\section{B. - Evolution CHEZ Le RONGeur.}

Le développement des larves puis des adultes chez l'hôte définitif est obtenu en infestant par voie buccale de jeunes Apodemus sylvaticus. Ceux-ci, capturés à la Borie Nouvelle, sont préalablement maintenus en captivité depuis quinze jours afin d'éviter toute infestation spontanée par de jeunes stades larvaires. Cette étude est complétée par l'infestation de souris de laboratoire. L'évolution du Nématode chez le Rongeur est résumée dans le tableau p. 98. Les dimensions sont en microns à l'exception de la taille des femelles mûres exprimée en $\mathrm{mm}$.

Troisième mue: La troisième mue de Rictularia proni a lieu entre $48 \mathrm{~h}$ et $72 \mathrm{~h}$ chez l'hôte définitif. Ce passage très rapide du troisième au quatrième stade larvaire est mis en évidence par la brusque augmentation de taille entre les larves de $48 \mathrm{~h}$ et de $72 \mathrm{~h}$, par la mue, observée après un séjour de plusieurs heures dans du milieu Vago, des larves de 48 h (fig. $3 \mathrm{C}$ ), enfin, par les structures céphaliques différentes des larves âgées de trois jours.

Les larves du quatrième stade conservent par ailleurs l'aspect général des larves du troisième stade.

Quatrième mue: La durée du développement chez l'hôte définitif paraît plus brève chez le mâle que chez la femelle, car quatre jours après l'infestation un mâle orné de 40 paires d'éléments cuticulaires est récolté dans les villosités intestinales. Cette ornementation caractérise en effet les adultes mâles.

En dehors de la taille, aucune différence morphologique n'apparaît entre les larves femelles âgées de $72 \mathrm{~h}$, de $96 \mathrm{~h}$ et de $112 \mathrm{~h}$. Seules, celles de $112 \mathrm{~h}$, placées dans du milieu Vago se débarassent de leur exuvie (fig. 4 C). 


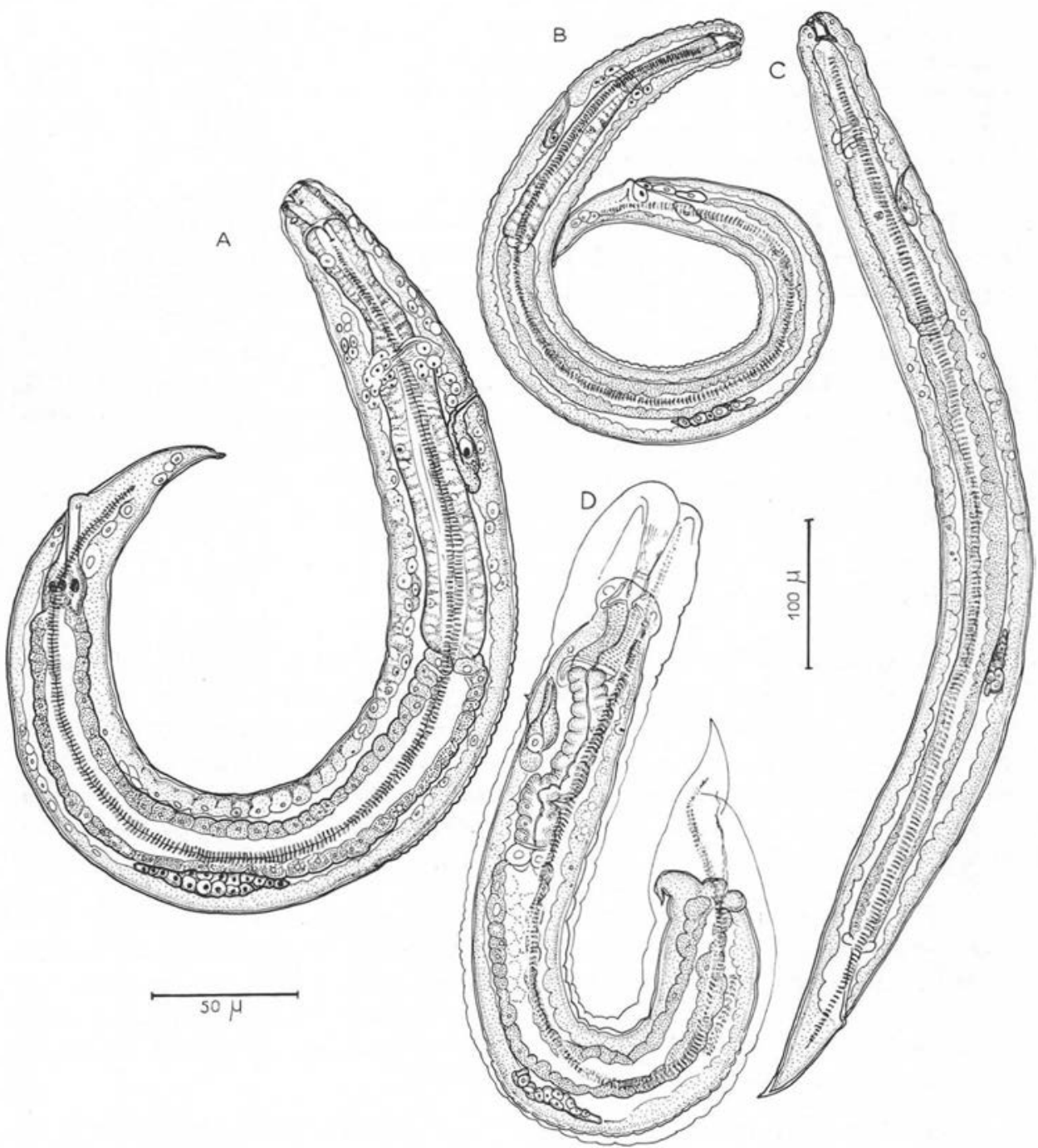

Fig. 3. $-3^{e}$ stade larvaire et $3^{\circ}$ mue. A : larve infestante chez l'Insecte, vue latérale; B : larve du $3^{\text {c }}$ stade après $24 \mathrm{~h}$ de croissance chez le Rongeur, vue latérale; C: larve du $3^{\text {e }}$ stade après $48 \mathrm{~h}$ de croissance chez le Rongeur, vue latérale; D : $3^{\circ}$ mue obtenue sur les larves de $48 \mathrm{~h}$ placées dans du milieu Vago

$$
\text { A : éch. } 50 \mu ; \mathrm{B}, \mathrm{C} \text { et D : éch. } 100 \mu
$$

Une autopsie réalisée $142 \mathrm{~h}$ après l'infestation permet de récolter $1 \sigma^{*}$ et 2 \% adultes. Les premiers œufs, renfermant des larves du premier stade, sont observés trente jours après l'infestation dans l'utérus des femelles. 


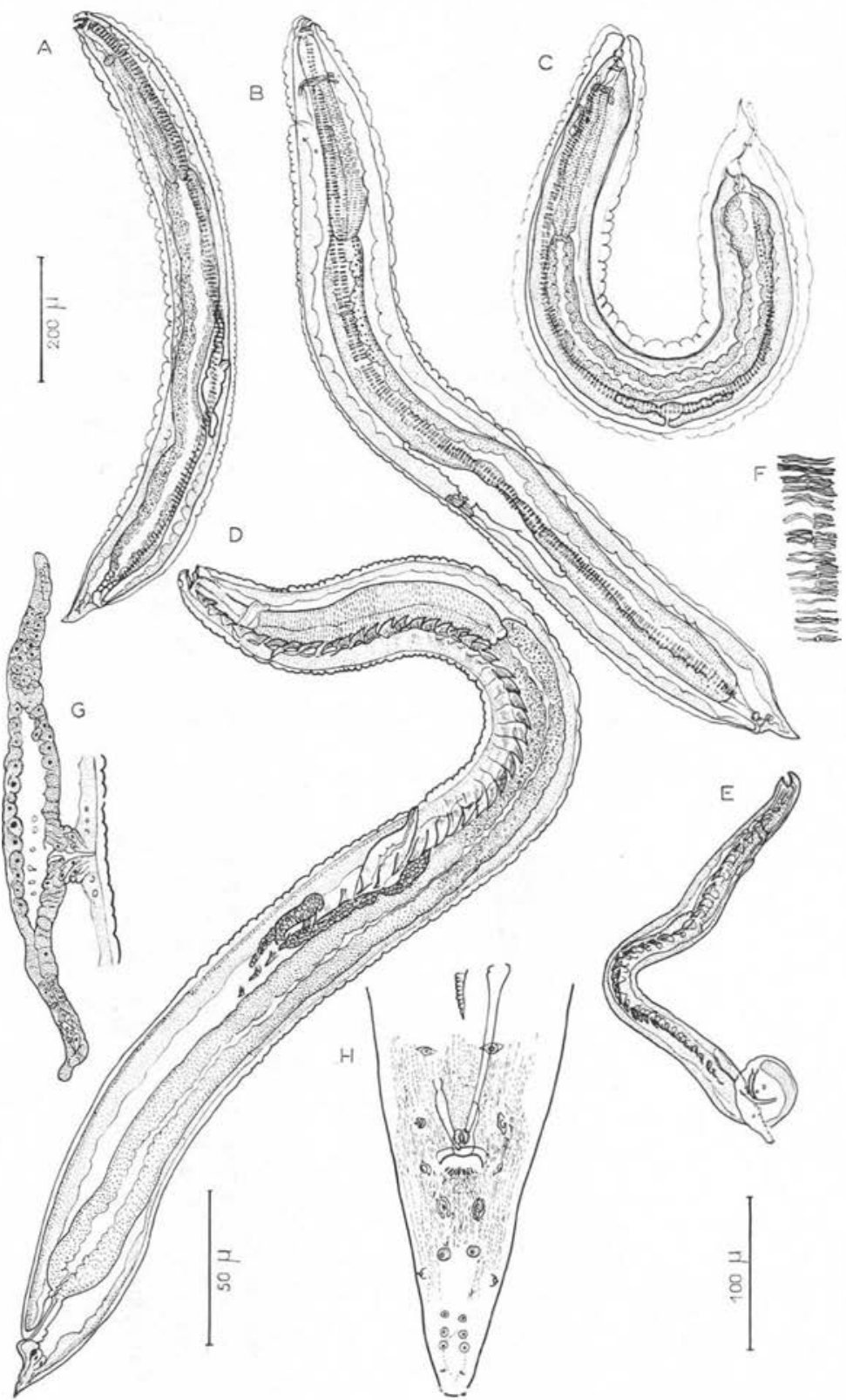

FIG. 4. $-4^{*}$ stade, $4^{*}$ mue et adultes. A : larve $q$ après trois jours d'évolution chaz le Rongeur; B : larve $\Varangle$ âgée de $112 \mathrm{~h} ; \mathrm{C}: 4^{\circ}$ mue; D: jeune $\bigcirc$ âgée d'à peine six jours; E: jeune $\delta^{\star}$ récolté quatre jours après l'infestation ; $F$ : détail d'une aile latérale du $4^{*}$ stade; $G$ : ébauche génitale $q$ du $4^{\circ}$ stade de $112 \mathrm{~h} ; \mathrm{H}$ : bourse caudale d'un $\hat{\delta}$ âgé de trente jours, vue ventrale, l'ornementation précloacale comporte cinq à sept arêtes cuticulaires impaires

A, B, C, D, E : éch. $200 \mu ;$ F : éch. $100 \mu ;$ G, H : éch. $50 \mu$ 


\begin{tabular}{|c|c|c|c|c|c|c|c|c|c|c|c|c|c|c|c|c|c|}
\hline \multirow{5}{*}{$\frac{\text { ڤ్ }}{3}$} & \multirow{2}{*}{$\stackrel{\text { घ் }}{\Sigma}$} & \multirow{2}{*}{$\stackrel{\infty}{\stackrel{0}{\Xi}}$} & \multirow[b]{2}{*}{$\cong$} & $\begin{array}{l}0+ \\
0\end{array}$ & $\begin{array}{l}E \\
\text { E } \\
\infty \\
\dot{+}\end{array}$ & 8 & 胥 & ह్రి & : & ્ㅗ & ำ & $\begin{array}{l}8 \\
n \\
n \\
n\end{array}$ & $\begin{array}{l}E \\
\Xi \\
\dot{Z}\end{array}$ & & ำ & & \\
\hline & & & & 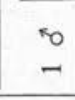 & $\begin{array}{l}\stackrel{0}{\circ} \\
\alpha \\
\dot{\gamma}\end{array}$ & กิ & 요 & 윰 & ঙ્ণ & $\dddot{q}$ & 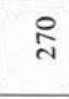 & 움 & $\begin{array}{l}0 \\
n \\
\infty \\
m\end{array} \mid$ & ¿ & 욤 & & $\stackrel{+}{+}$ \\
\hline & & & & & & & & & & & & & & & & & \\
\hline & \multirow{2}{*}{$\stackrel{\dot{m}}{<}$} & \multirow{2}{*}{$\begin{array}{l}\text { I } \\
\text { ำ }\end{array}$} & \multirow{2}{*}{$\infty$} & $\begin{array}{l}\mathrm{a} \\
\mathrm{d}\end{array}$ & $\frac{d}{d}$ & $\cong$ & 웜 & ڤิ & ㅇ & $\stackrel{\sim}{N}$ & $\cong$ & $\stackrel{9}{7}$ & 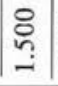 & 产 & $\stackrel{9}{9}$ & $\begin{array}{l}= \\
8\end{array}$ & \\
\hline & & & & "0 & $\begin{array}{l}\text { ำ } \\
\text { ñ }\end{array}$ & $\infty$ & $\cong$ & $\infty$ & ㅇ & $\stackrel{\sim}{\sim}$ & $\infty$ & 원 & ஓ్ & & & 8 & ㅇํำ \\
\hline $\begin{array}{l}\stackrel{0}{\text { हี }} \text { o+ } \\
\stackrel{8}{+}\end{array}$ & & & & & & & & & & & & & & & & & \\
\hline \multirow{3}{*}{ 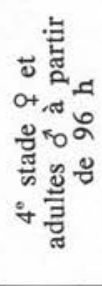 } & $\stackrel{\dot{q}}{<}$ & $\stackrel{\text { I }}{\stackrel{\text { J }}{\beth}}$ & $\infty$ & 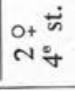 & $\frac{0}{n}$ & 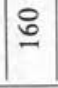 & $\cong$ & $\cong$ & $\stackrel{\infty}{\infty}$ & 이 & ి્న & $\stackrel{n}{2}$ & @્ & & 용 & 8 & \\
\hline & \multirow{2}{*}{$\stackrel{\dot{q}}{4}$} & \multirow{2}{*}{ 둥 } & \multirow{2}{*}{$\infty$} & $\begin{array}{l}\text { of } \dot{\text { के }} \\
\text { mit }\end{array}$ & 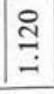 & $\stackrel{ }{=}$ & $\cong$ & in & \& & $\vec{\sim}$ & $\approx$ & & 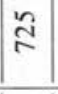 & & 六 & $\stackrel{2}{r}$ & \\
\hline & & & & ○胥 & $\stackrel{\circ}{\circ}$ & 8 & 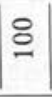 & $\stackrel{0}{n}$ & $\cong$ & $\approx$ & $\infty$ & $\cong$ & ถู & & \& & ㅇ & $n^{R}$ \\
\hline \multicolumn{18}{|l|}{ 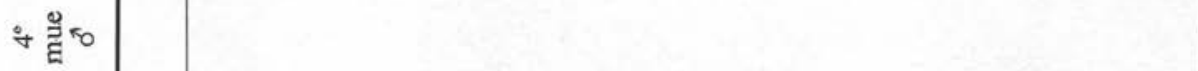 } \\
\hline 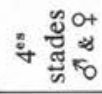 & $\stackrel{\dot{q}}{\dot{q}}$ & $\frac{\pi}{1}$ & $\infty$ & $m$ & 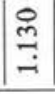 & $\cong$ & ฉ̊ & $\stackrel{\circ}{2}$ & $\stackrel{ }{2}$ & 2 & $\stackrel{R}{R}$ & สิ & $\stackrel{1}{1}$ & 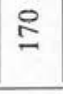 & $\tilde{f}$ & $\approx$ & \\
\hline \multicolumn{18}{|l|}{ in } \\
\hline \multirow{2}{*}{$\begin{array}{l}\text { वँّ } \\
\text { in } \\
\text { in }\end{array}$} & $\stackrel{\dot{q}}{\dot{\psi}}$ & $\stackrel{5}{\infty}$ & $\infty$ & 0 & ㅇำ & 8 & 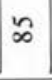 & $\stackrel{\text { తి }}{=}$ & I & $\stackrel{\bullet}{-}$ & $\stackrel{2}{1}$ & 足 & 암 & $\vartheta$ & $\frac{n}{m}$ & $\approx$ & \\
\hline & $\stackrel{\dot{\varphi}}{\dot{\varphi}}$ & 咅 & $\infty$ & $m$ & $\underset{ }{T}$ & 음 & $\bar{\infty}$ & ల్లి & 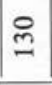 & \pm & 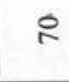 & 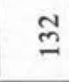 & $\dddot{z}$ & 8 & กี & $\mathscr{\infty}^{\mathbb{2}}$ & \\
\hline 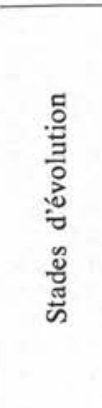 & 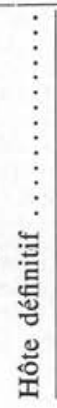 & 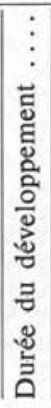 & 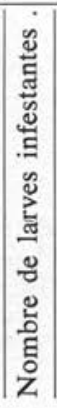 & 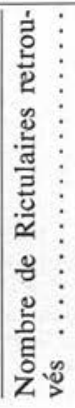 & \begin{tabular}{|c}
$\vdots$ \\
$\vdots$ \\
$\vdots$ \\
$\vdots$ \\
$\vdots$ \\
$\vdots$ \\
$\vdots$ \\
0 \\
$\vdots$ \\
5 \\
$\vdots$
\end{tabular} & 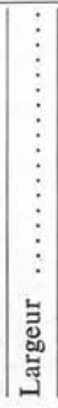 & 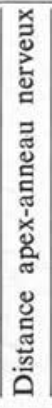 & 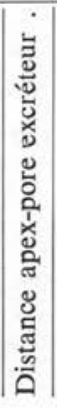 & 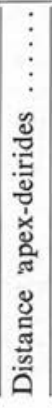 & 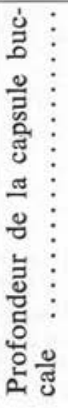 & 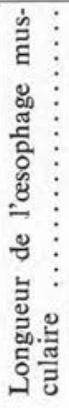 & 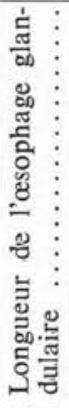 & 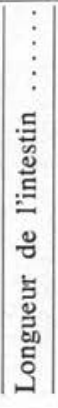 & 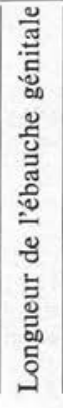 & 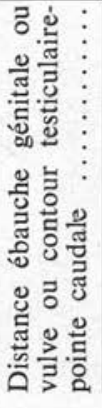 & 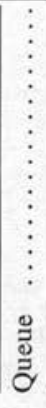 & 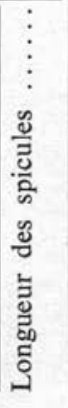 \\
\hline
\end{tabular}




\section{Ontogénèse des structures céphaliques}

Premier stade: (fig. 5 I). L'épine cuticulaire subapicale de la larve du premier stade est réduite, elle est soulignée à sa base d'un fin repli.

Deuxième stade: (fig. 5 II). L'ouverture buccale ovale est allongée dorso-ventralement. Elle est entourée d'un premier cycle de six minuscules papilles et d'un second de six autres terminaisons plus importantes. La section du vestibule buccal triangulaire dans la partie supérieure devient étroite et circulaire en profondeur.

Troisième stade chez l'Insecte: (fig. 5 III $a$ ). La bouche est légèrement déportée dorsalement. Il en résulte une dissymétrie des bordures dorsale et ventrale.

Les six papilles latérales internes ne sont pas réunies par des ornementations cuticulaires; les deux dorsales sont accolées. Les terminaisons sensorielles céphaliques comptent en outre deux amphides et un cycle labial externe de quatre papilles rapprochées des quatre céphaliques.

Les pointes des trois dents œsophagiennes sont orientées vers la région dorsale.

Troisième stade chez le Rongeur: (fig. $5 \mathrm{III} b, c$ ). Ces déformations évolutives s'accentuent après $24 \mathrm{~h}$, puis $48 \mathrm{~h}$ de développement chez l'hôte définitif: les deux dents latéro-ventrales sont dirigées au-dessus de la dent dorsale qui compte deux à trois denticules, la bouche émigre vers la région dorsale de l'apex.

Quatrième stade: (fig. 5 IV). Les larves du quatrième stade apparues après trois jours d'évolution chez le Rongeur diffèrent essentiellement de celles du troisième stade par leur structure céphalique.

Les deux dents œsophagiennes denticulées ventrales dont les vestiges après la mue, subsistent retenus au fond de la capsule, disparaissent sur les quatrièmes stades femelles de quatre jours.

Elles font place à une voûte buccale continue dont le bord ventral est orné de huit à dix denticules.

- La dent œsophagienne ventrale denticulée du troisième stade est remplacée par une apophyse arrondie qui préfigure l'épaisse dent dorsale de l'adulte.

- Le bord ventral de la capsule buccale est orné de cinq à six denticules.

- L'ouverture buccale d'une larve de quatre jours compte seize denticules.

Adultes: (fig. 5 V). Après une évolution de six jours chez le Mulot, le Nématode est adulte. La morphologie de la capsule buccale du mâle et de la femelle est définitive. Sa paroi ventrale est fortement inclinée sur la paroi dorsale d'où fait saillie, à la base, l'unique dent œsophagienne. Le pourtour buccal de la femelle compte alors 26 denticules, nombre qui ne variera que très faiblement chez les femelles gravides.

Chez le mâle, les denticules buccaux sont au nombre de 19 (fig. $5 \mathrm{~V} \mathrm{~b}$ ).

Chez les individus plus âgés, l'épaisseur relative du «casque musculaire » qui surmonte la capsule s'accroît entraînant la migration des papilles du cycle labial interne vers le dos. 


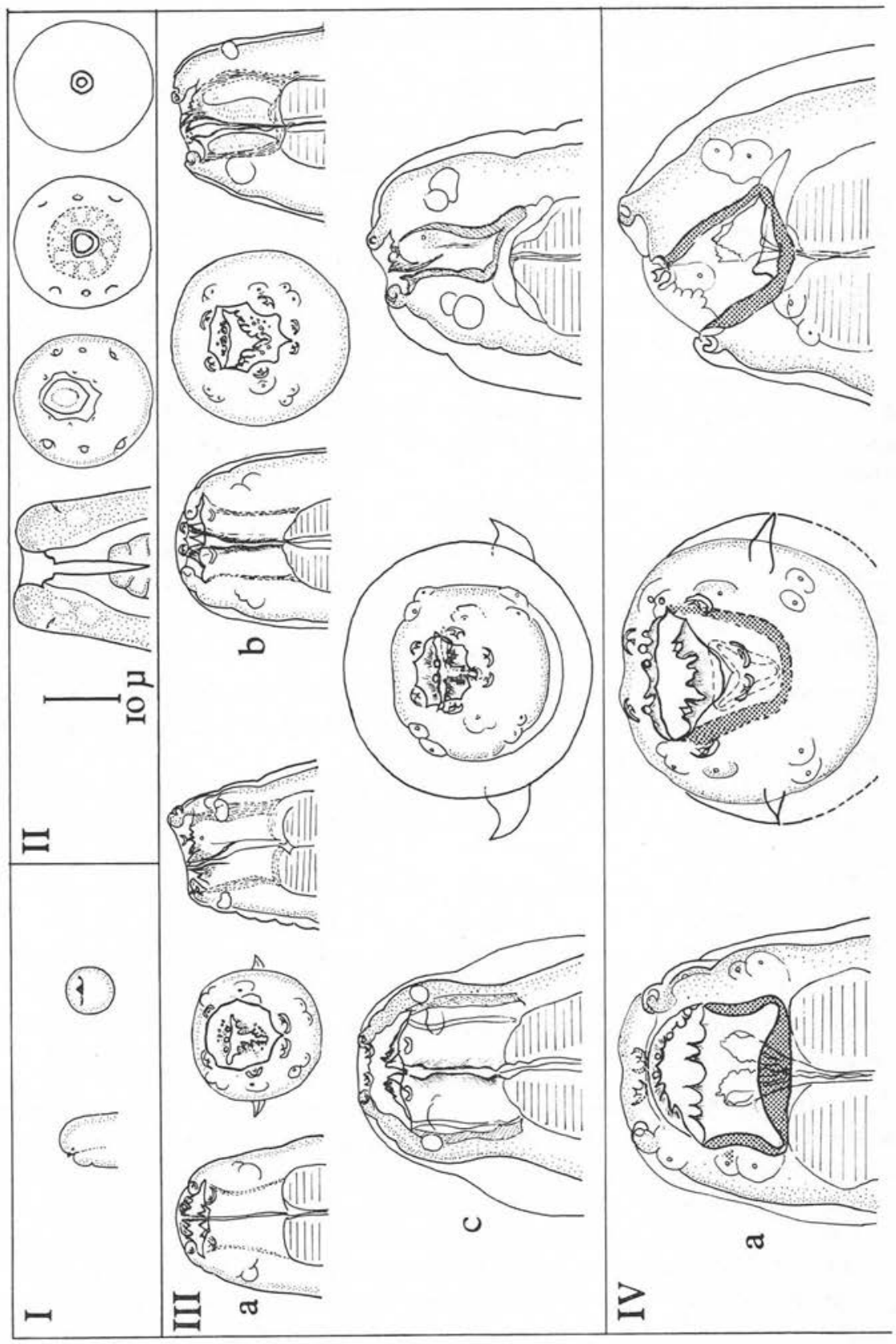




\section{Conclusion}

L'évolution de Rictularia proni Seurat, 1915, chez l'Insecte, puis chez le Rongeur présente un double intérêt biologique et phylogénétique.

\section{A. - INTÉRÊt BIOLOGIQUe.}

1) Morphologie larvaire: La taille des formes infestantes de $R$. proni dépasse rarement $500 \mu$ de long. Ces larves sont donc différenciables de celles de l'espèce la plus proche $R$. cristata Frœlich, 1802 dont les larves du $3^{\circ}$ stade ont une longueur comprise entre 670 et $896 \mu$ (cf. Morosov, 1960).

Cette taille réduite chez une larve de Spiruride confirme l'identification de Rioux, et coll. d'une larve du troisième stade de $R$. proni infestant spontanément Phlebotomus ariasi Tonnoir.

2) La spécificité au niveau de l'hôte intermédiaire est faible puisque le parasite peut évoluer chez des Insectes appartenant à des Ordres divers : Diptères, Orthoptères, Dermaptères, Coléoptères.

3) L'évolution de Rictularia proni se déroule selon des normes précises qui confirment les observations relevées au cours des autres cycles de Rictulariidae.

Le développement de ces Nématodes est en effet très rapide. Chez l'Insecte maintenu à une température de 25 à $28^{\circ} \mathrm{C}$ la larve est au troisième stade vers le treizième jour et entre en diapause. La brieveté des troisième et quatrième stades larvaires caractérise l'évolution chez l'hôte définitif. Le quatrième stade apparaît après 48 heures chez le Rongeur. Les mâles et les femelles sont adultes avant le sixième jour.

\section{B. - INTÉRÊT PHYLOGÉNÉtiQUe.}

Dans la famille des Rictulariidas, la séparation entre les genres Rictularia Froelich, 1802 et Pterygodermatites Wedl, 1861 que nous avons principalement caractérisée par la morphologie des structures apicales des adultes, se réalise très tôt lors de l'ontogénèse des structures céphaliques de $R$. proni.

Dans le genre Pterygodermatites les structures apicales des larves du troisième stade ont, en effet été étudiées chez deux espèces.

- P. zygodontomis (Quentin, 1967) (espèce néotropicale) : la bouche est apicale, hexagonale, garnie de trois petites dents œsophagiennes. Les papilles du cycle labial interne sont simples sans ornementation cuticulaire.

- P. desportesi (Chabaud et Rousselot, 1956) (espèce éthiopienne) : la tête de la larve porte une capsule terminale, des papilles labiales internes réunies entre elles par des cordons d'ornementation cuticulaire, trois dents œsophagiennes d'égale importance et denticulées. Ces caractères céphaliques ne correspondent pas à l'adulte du parasite, mais rappellent ceux de certaines espèces néarctiques les plus primitives. 
- Le troisième stade de Rictularia proni chez l'hôte intermédiaire présente déjà une ouverture buccale et des dents œsophagiennes inclinées dorsalement, ce qui affecte la symétrie dorso-ventrale de la bouche.

- La séparation générique devient totale dès le quatrième stade chez l'hôte définitif par la perte des trois dents œsophagiennes denticulées, la néoformation de structures buccales différentes qui confirment l'apparition d'une lignée évolutive distincte dans cette famille de Spiruride hautement diversifiée.

\section{Bibliographie}

Morosov (J. F.), 1960. - Sur la biologie du cycle de Rictularia amurensis Schulz, 1927 (Nematoda Rictulariidae) (En Russe). Inst. Ped. Gork., 27, sér. Helm., (2). p. 1728, fig. 1-9.

Quentin (J.-C.), 1969. - Essai de classification des Nématodes Rictulaires. Mém. Mus. Nat. Hist. Nat., nlle sér., sér. A. Zool., 54 (2), p. 55-115, fig. 1-27.

—, 1969. - Cycle biologique de Pterygodermatites desportesi (Chabaud et Rousselot, 1956), Nematoda Rictulariidae. Ann. Parasit. Hum. Comp., 44 (1), p. 47-48, fig. 1-6.

Rioux (J.-A.), Сhabaud (A.-G.), Bain (O.), Quentin (J.-C.) et Desset (M,-C.), - Infestation spontanée de Phlebotomus ariasi par Rictularia proni, Spiruride parasite de Mulots; Les terriers de Mulots sont-ils des gîtes larvaires à Phlebotomus? Ibid. (sous presse).

Seurat (L.-G.), 1915. - Sur les Rictulaires des Carnivores du Nord-Africain et les affinités du genre Rictularia. C.R. Soc. Biol., 78 (11), p. 318-322, fig. 1-3.

-, 1916. - Sur l'habitat normal et les affinités du Rictularia proni Seur. C.R. Soc. Biol.. 79 (3), p. 146-149, fig. 1-2. 